\title{
A Nonlinear Macrodynamic Model with Fixed Exchange Rates: Its Dynamics and Noise Effects
}

\author{
TOICHIRO ASADA ${ }^{\mathrm{a}, *}$, TOSHIO INABA ${ }^{\mathrm{b}}$ and TETSUYA MISAWA ${ }^{\mathrm{c}}$ \\ ${ }^{a}$ Faculty of Economics, Chuo University, 742-1, Higashinakano, Hachioji, Tokyo 192-0393, Japan; ${ }^{b}$ School of Education, \\ Waseda University, 1-6-1, Nishiwaseda, Shinjuku-ku, Tokyo 169-0051, Japan; ${ }^{\mathrm{c}}$ Faculty of Economics, \\ Nagoya City University, Mizuho-cho, Mizuho-ku, Nagoya 467-0001, Japan
}

(Received 21 October 1998; In final form 20 June 1999)

\begin{abstract}
In this paper, we formulate a discrete time version of the Kaldorian macrodynamic model in a small open economy with fixed exchange rates. The model is described by a system of the three-dimensional nonlinear difference equations with and without stochastic disturbances (noise effects). We study the local stability/instability properties analytically by using the linear approximation method, and chaotic dynamics with and without noise effects are investigated by means of numerical simulations. In general, it is believed that the effect of the noise is to obscure the basic structure of the system. But, this is not necessarily the case. We show by means of numerical analysis that the noise can reveal the hidden structure of the model contrary to the usual intuition in some situations.
\end{abstract}

Keywords: Fixed exchange rates, Noise effects, Nonlinear macrodynamics, Small open economy

\section{INTRODUCTION}

The purpose of this paper is to formulate a macrodynamic model which is described by a system of the three-dimensional nonlinear difference equations with and without stochastic disturbances (noise effects), and to investigate its behavior by means of analytical method and numerical simulations. The model presented in this paper is a discrete time version of the Kaldorian business cycle model in an open economy which was formulated by
Asada (1995) as a continuous time model. ${ }^{\dagger}$ Contrary to Asada (1995)'s original model, we introduce the noise effects.

Generally speaking, the economy is not isolated system, but it is subject to the interactions with other subsystems of the society. One of the effective methods to model such influences is to introduce the 'noise' (stochastic disturbance). In our model, it is supposed that another subsystem named 'foreign country' exists outside the system, and the dynamics of the economy are affected by the

\footnotetext{
* Corresponding author. E-mail: asada@tamacc.chuo-u.ac.jp.

${ }^{\dagger}$ The original version of the Kaldorian business cycle model in a closed economy was presented by Kaldor (1940)'s classical paper, and it was later refined by several authors. See, for example, Chang and Smyth (1971), Gabisch and Lorenz (1989), and Lorenz (1993).
} 
transactions with 'foreign country'. We assume that the parameter $\beta$ which reflects the 'degree of capital mobility' is subject to the stochastic disturbances, and study the effects of the noise on the dynamics of the system by means of numerical simulations.

A seminal paper which introduced noise into the Kaldorian business cycle model in a closed economy is Kosobad and O'Nell (1972)'s model. Dohtani et al. (1996)'s work is a more recent contribution. In particular, Dohtani et al. (1996) introduced the noise effects into the Kaldorian business cycle model which is described by the two-dimensional nonlinear difference equations, and showed by means of numerical experimentation that the noise can reveal rather than obscure the hidden structure of the system in some situations contrary to the usual intuition. In this paper, we show that such a conclusion also applies in an extended version of the three-dimensional Kaldorian system in an open economy with fixed exchange rates. In particular, it is shown by means of numerical approach that the noise can reveal the hidden chaotic attractors at the vicinity of the 'window', and two separate chaotic attractors can be combined under the influence of the noise.

\section{THE MODEL}

Asada (1995) tried to extend the Kaldorian type of the nonlinear business cycle model to the small open economy by using the deterministic continuous time model. In Asada (1995), both the system of fixed exchange rates and that of flexible exchange rates were formulated and investigated by analytical method and numerical simulations. In particular, the effect of the change of the parameter $\beta$ which represents the 'degree of capital mobility' were analyzed, and it was shown by means of the Hopf bifurcation theorem that the cyclical fluctuation can occur at some parameter values in the system of fixed exchange rates.
In this paper, we shall consider a stochastic version of Asada (1995)'s model of fixed exchange rates. $^{\ddagger}$ We can describe the basic system of equations as follows:

$$
\begin{gathered}
Y_{t+1}-Y_{t}=\alpha\left[C_{t}+I_{t}+G+J_{t}-Y_{t}\right], \alpha>0 ; \\
K_{t+1}-K_{t}=I_{t} ; \\
C_{t}=c\left(Y_{t}-T_{t}\right)+C_{0} ; \quad 0<c<1, C_{0}>0 ; \\
I_{t}=I\left(Y_{t}, K_{t}, r_{t}\right) ; \quad I_{Y} \equiv \partial I_{t} / \partial Y_{t}>0 \\
I_{K} \equiv \partial I_{t} / \partial K_{t}<0, I_{r} \equiv \partial I_{t} / \partial r_{t}<0 ; \\
T_{t}=\tau Y_{t}-T_{0} ; \quad 0<\tau<1, T_{0}>0 ; \\
M_{t} / p=L\left(Y_{t}, r_{t}\right) ; \quad L_{Y} \equiv \partial L_{t} / \partial Y_{t}>0 \\
L_{t} \equiv \partial L_{t} / \partial r_{t}<0 \\
J_{t}=J\left(Y_{t}, E_{t}\right) ; \quad J_{Y} \equiv \partial J_{t} / \partial Y_{t}<0 \\
J_{E} \equiv \partial J_{t} / \partial E_{t}>0 \\
Q_{t}=\left(\beta+\sigma \gamma_{t}\right)\left\{r_{t}-r_{f}-\left(E_{t}^{e}-E_{t}\right) / E_{t}\right\} \\
\beta>0, \sigma \geq 0 ;
\end{gathered}
$$

where the meanings of the symbols are as follows: $Y=$ net real national income, $C=$ real consumption expenditure, $I=$ net real private investment expenditure, $G=$ real government expenditure (fixed), $K=$ real physical capital stock, $T=$ real income tax, $M=$ nominal money supply, $p=$ price level

\footnotetext{
${ }^{\ddagger}$ The model of flexible exchange rates will be considered separately in another paper.
} 
(fixed), $r=$ nominal domestic rate of interest, $r_{\mathrm{f}}=$ nominal foreign rate of interest (fixed), $E=$ value of a unit of foreign currency in terms of domestic currency (exchange rate), $E^{\mathrm{e}}=$ expected exchange rate of near future, $J=$ balance of current account (net export) in real terms, $Q=$ balance of capital account in real terms, $A=$ total balance of payments in real terms, $\alpha=$ adjustment speed in the goods market, $\beta=$ parameter which represents the "degree of capital mobility" $(\beta>0), \gamma=$ normal pseudo-random number $N(0,1), \sigma=$ standard deviation parameter $(\sigma \geq 0)$. The subscript $t$ denotes time period.

Equation (1) formulates the quantity adjustment process in the goods market, i.e., $Y_{t}$ fluctuates according as the excess demand in the goods market is positive or negative. Equation (2) is the capital accumulation equation. Equations (3)-(5) are consumption function, investment function, and income tax function respectively. Equation (6) is the equilibrium condition in the money market. Equation (7) is the current account function. Equation (8) says that the balance of capital account depends on the difference between the rates of return of domestic and foreign bonds. It is assumed that the parameter $\beta$ (degree of capital mobility) is fluctuated by noise. Equation (9) is the definition of the total balance of payments. Equations (10) and (11) express the institutional arrangement of the system of fixed exchange rates. Equation (12) says that money supply endogenously fluctuates according as the total balance of payments is positive or negative under the system of fixed exchange rates.

These equations can be reduced to the following set of three-dimensional nonlinear difference equations: ${ }^{\text {I }}$

(i) $Y_{t+1}=Y_{t}+\alpha\left[c(1-\tau) Y_{t}+c T_{0}+C_{0}\right.$

$$
\begin{aligned}
& +G+I\left(Y_{t}, K_{t}, r\left(Y_{t}, M_{t}\right)\right) \\
& \left.+J\left(Y_{t}, \bar{E}\right)-Y_{t}\right] \\
\equiv & F_{1}\left(Y_{t}, K_{t}, M_{t} ; \alpha\right)
\end{aligned}
$$

(ii) $K_{t+1}=K_{t}+I\left(Y_{t}, K_{t}, r\left(Y_{t}, M_{t}\right)\right)$

$$
\equiv F_{2}\left(Y_{t}, K_{t}, M_{t}\right) \text {; }
$$

(iii)

$$
\begin{aligned}
M_{t+1}= & M_{t}+p J\left(Y_{t}, \bar{E}\right) \\
& +\left(\beta+\sigma \gamma_{t}\right) p\left\{r\left(Y_{t}, M_{t}\right)-r_{f}\right\} \\
\equiv & F_{3}\left(Y_{t}, M_{t} ; \beta, \sigma\right) .
\end{aligned}
$$

We shall call the system $\left(\mathrm{S}_{1}\right)$ 'model 1'. By the way, Chang and Smyth (1971)'s version of the Kaldorian business cycle model adopts the following type of the saving function:

$$
\begin{aligned}
& S_{t}=S\left(Y_{t}, K_{t}\right) ; \quad 1>S_{Y} \equiv \partial S_{t} / \partial Y_{t}>0 \\
& S_{K} \equiv \partial S_{t} / \partial K_{t}<0 .
\end{aligned}
$$

Since the saving $S_{t}$ is the difference between the disposable income and the consumption $C_{t}$, Eq. (13) implies the following type of the consumption function:

$$
\begin{gathered}
C_{t}=C\left(Y_{t}, K_{t}\right) ; \quad 1>C_{Y} \equiv \partial C_{t} / \partial Y_{t}>0 \\
C_{K} \equiv \partial C_{t} / \partial K_{t}>0 .
\end{gathered}
$$

This consumption function represents a sort of the 'wealth effect', i.e., the increase of the real capital stock stimulates the consumption expenditure. If we adopt this type of consumption function, we must replace Eq. $\left(S_{1}\right)$-(i) with the following equation:

$$
\begin{aligned}
Y_{t+1}= & Y_{t}+\alpha\left[C\left(Y_{t}, K_{t}\right)+I\left(Y_{t}, K_{t}, r\left(Y_{t}, M_{t}\right)\right)\right. \\
& \left.+G+J\left(Y_{t}, \bar{E}\right)-Y_{t}\right] .
\end{aligned}
$$

In this case, we have

$$
\begin{gathered}
\frac{\partial Y_{t+1}}{\partial K_{t}}=\alpha\left(C_{K}+I_{K}\right)>\alpha I_{K} . \\
(+) \quad(-)(-)
\end{gathered}
$$

In particular, in the special case of $C_{K}=\left|I_{K}\right|$, we obtain

$$
\frac{\partial Y_{t+1}}{\partial K_{t}}=0
$$

\footnotetext{
`The expression $r\left(Y_{t}, M_{t}\right)$ is the 'LM equation' which is derived from Eq. (6). It is easy to see that $r_{Y} \equiv \partial r_{t} / \partial Y_{t}>0$ and $r_{M} \equiv$ $\partial r_{t} / \partial M_{t}<0$.
} 
In other words, the negative effect of the change of $K_{t}$ on $Y_{t+1}$ through the negative effect on the investment expenditure tends to be canceled out by the positive effect on the consumption expenditure when the 'wealth effect' exists. If Eq. (17) is satisfied, the system $\left(\mathrm{S}_{1}\right)$ must be modified as follows:

$$
\begin{aligned}
\text { (i) } \quad Y_{t+1}=F_{1}\left(Y_{t}, M_{t} ; \alpha\right) ; \\
\text { (ii) } \quad K_{t+1}=F_{2}\left(Y_{t}, K_{t}, M_{t}\right) ; \\
\text { (iii) } \quad \mathrm{M}_{t+1}=F_{3}\left(Y_{t}, M_{t} ; \beta, \sigma\right) .
\end{aligned}
$$

In this system, $Y_{t+1}$ is independent of $K_{t}$ so that the system becomes 'decomposable'. In other words, the path of $K_{t}$ depends on the paths of $Y_{t}$ and $M_{t}$, but the movements of $Y_{t}$ and $M_{t}$ are independent of the path of $K_{t}$. We shall refer to the system $\left(\mathrm{S}_{2}\right)$ as 'model 2'.

\section{LOCAL STABILITY-INSTABILITY ANALYSIS OF 'MODEL 1'}

First, let us consider the local stability-instability analysis of 'model 1' by assuming $\sigma=0$ (no stochastic disturbance). Asada (1995) proved that the system $\left(\mathrm{S}_{1}\right)$ has the unique equilibrium point $\left(Y^{*}, K^{*}, M^{*}\right)>(0,0,0)$ under some reasonable conditions. In this paper, we shall assume that such an equilibrium point in fact exists. The Jacobian matrix of this system which is evaluated at the equilibrium point can be written as follows:

$$
J_{1}=\left[\begin{array}{ccc}
F_{11}(\alpha) & F_{12}(\alpha) & F_{13}(\alpha) \\
F_{21} & F_{22} & F_{23} \\
F_{31}(\beta) & 0 & F_{33}(\beta)
\end{array}\right],
$$

where

$$
\begin{array}{r}
F_{11}(\alpha)=1+\alpha\left[\begin{array}{c}
\left.\alpha I_{Y}+I_{r} r_{Y}-\{1-c(1-\tau)+m\}\right], \\
(+)(-)(+)
\end{array}\right. \\
F_{12}(\alpha)=\underset{(-)}{\alpha I_{K}<0,} \quad F_{13}(\alpha)=\underset{(-)}{\alpha I_{r} r_{M}>0,}, \\
(-)(-)
\end{array}
$$

$$
\begin{aligned}
& F_{21}=I_{Y}+I_{r} r_{Y}, F_{22}=1+I_{K}, F_{23}=I_{r} r_{M}>0 \text {, } \\
& (+)(-)(+) \quad(-) \quad(-)(-) \\
& F_{31}(\beta)=p\left(-m+\beta r_{Y}\right), \\
& F_{33}(\beta)=1+\beta p r_{M} \text {. } \\
& (-)
\end{aligned}
$$

In these expressions, $m=-J_{Y}>0$ is the 'marginal propensity to import'. The characteristic equation of this system is expressed as

$$
\begin{aligned}
\Delta_{1}(\lambda) & =\left|\lambda I-J_{1}\right| \\
& =\lambda^{3}+a_{1} \lambda^{2}+a_{2} \lambda+a_{3}=0,
\end{aligned}
$$

where

$$
a_{1}=-\operatorname{trace} J_{1}=-F_{11}(\alpha)-F_{22}-F_{33}(\beta),
$$

$$
\begin{aligned}
a_{2}= & \left|\begin{array}{cc}
F_{22} & F_{23} \\
0 & F_{33}(\beta)
\end{array}\right|+\left|\begin{array}{cc}
F_{11}(\alpha) & F_{13}(\alpha) \\
F_{31}(\beta) & F_{33}(\beta)
\end{array}\right| \\
& +\left|\begin{array}{cc}
F_{11}(\alpha) & F_{12}(\alpha) \\
F_{21} & F_{22}
\end{array}\right| \\
= & F_{22} F_{33}(\beta)+F_{11}(\alpha) F_{33}(\beta)-F_{13}(\alpha) F_{31}(\beta) \\
& +F_{11}(\alpha) F_{22}-F_{12}(\alpha) F_{21},
\end{aligned}
$$

$$
\begin{aligned}
a_{3}= & -\operatorname{det} J_{1} \\
= & -F_{11}(\alpha) F_{22} F_{33}(\beta)-F_{12} F_{23} F_{31}(\beta) \\
& +F_{13}(\alpha) F_{22} F_{31}(\beta)+F_{12}(\alpha) F_{21} F_{33}(\beta) .
\end{aligned}
$$

The Cohn-Schur conditions for local stability can be expressed as follows: ${ }^{\S}$

$$
\begin{gathered}
1+a_{2}-\left|a_{1}+a_{3}\right|>0, \\
1-a_{2}+a_{1} a_{3}-a_{3}^{2}>0, \\
a_{2}<3 .
\end{gathered}
$$

\footnotetext{
$\S$ See Gandolfo (1996), p. 90. In fact, the condition (25) is redundant because this condition can be derived from other two conditions. However, for our purpose, this expression is convenient.
} 
From these local stability conditions we can derive a very simple sufficient condition for local instability, i.e., $a_{2}>3$. By using this local instability condition, we can derive the following proposition.

Proposition 1 Suppose that $I_{Y}<1-c(1-\tau)+$ $m$. Then, the equilibrium point of the system $\left(\mathrm{S}_{1}\right)$ is locally unstable if $\alpha>0$ and $\beta>0$ are sufficiently large.

Proof Differentiating Eq. (21), we have

$$
\begin{aligned}
& \partial a_{2} / \partial \alpha \\
&=\left\{F_{22}+F_{33}(\beta)\right\}\left\{\partial F_{11}(\alpha) / \partial \alpha\right\} \\
&-F_{31}(\beta)\left\{\partial F_{13}(\alpha) / \partial \alpha\right\}-F_{21}\left\{\partial F_{12}(\alpha) / \partial \alpha\right\} \\
&=\left(2 \beta+I_{K}+\beta p r_{M}\right)\left[I_{Y}+I_{r} r_{Y}\right. \\
&-\{1-c(1-\tau)+m\}] \\
&-p\left(-m+\beta r_{Y}\right) I_{r} r_{M}-\left(I_{Y}+I_{r} r_{Y}\right) I_{K} \\
&= \beta r_{M} p \underbrace{\left[I_{Y}-\{1-c(1-\tau)+m\}\right]}_{(-)} \\
&(-) \quad \\
&+p m-\left(I_{Y}+I_{r} r_{Y}\right) I_{K}+\left(2+I_{K}\right) \\
& \times\left[I_{Y}-\{1-c(1-\tau)+m\}+I_{r} r_{Y}\right] .
\end{aligned}
$$

From Eq. (26) we have $\lim _{\beta \rightarrow+\infty} \partial a_{2} / \partial \alpha=+\infty$ so that $\partial a_{2} / \partial \alpha$ becomes positive for sufficiently large $\beta>0$. In this case, $a_{2}>3$ for sufficiently large $\alpha$ and $\beta$.

Proposition 1 implies that under certain conditions, the increase of the adjustment speed in the goods market $(\alpha)$ and the degree of capital mobility $(\beta)$ tends to destabilize the system under the system of fixed exchange rates. This conclusion is in line with the result which was derived by Asada (1995)'s continuous time version of the model of fixed exchange rates.

\section{LOCAL STABILITY-INSTABILITY ANALYSIS OF 'MODEL 2'}

Next, we shall consider the local stability-instability analysis of 'model 2'. We also assume in this section that $\sigma=0$ (absence of noise effect). The
Jacobian matrix of the system $\left(\mathrm{S}_{2}\right)$ becomes

$$
J_{2}=\left[\begin{array}{ccc}
F_{11}(\alpha) & 0 & F_{13}(\alpha) \\
F_{21} & F_{22} & F_{23} \\
F_{31}(\beta) & 0 & F_{33}(\beta)
\end{array}\right],
$$

where the meanings of the symbols are the same as those of the previous chapter. The characteristic equation of this system is

$$
\Delta_{2}(\lambda)=\left|\lambda I-J_{2}\right|=\left(\lambda-F_{22}\right)\left(\lambda^{2}+b_{1} \lambda+b_{2}\right)=0,
$$

where

$$
\begin{gathered}
b_{1}=-F_{11}(\alpha)-F_{33}(\beta), \\
b_{2}=F_{11}(\alpha) F_{33}(\beta)-F_{13}(\alpha) F_{31}(\beta) .
\end{gathered}
$$

We can express the Cohn-Schur conditions for local stability as follows:"

$$
\begin{gathered}
\left|F_{22}\right|<1, \\
1+b_{2}>\left|b_{1}\right|, \\
b_{2}<1 .
\end{gathered}
$$

Equation (31) is equivalent to the condition $\left|I_{K}\right|<1$. We assume that in fact this condition is satisfied. By the way, we can easily see that $b_{2}>1$ is a sufficient condition for local instability.

Differentiating Eq. (30), we have

$$
\begin{aligned}
\partial b_{2} / \partial \alpha= & F_{33}(\beta)\left\{\partial F_{11}(\alpha) / \partial \alpha\right\} \\
& -F_{31}(\beta)\left\{\partial F_{13}(\alpha) / \partial \alpha\right\} \\
= & \beta r_{M} p \underbrace{\left[I_{Y}-\{1-c(1-\tau)\}+m\right]}_{(-)} \\
& (-) \quad p m+\left[I_{Y}-\{1-c(1-\tau)+m\}+I_{r} r_{Y}\right] .
\end{aligned}
$$

\footnotetext{
${ }^{\|}$See Okuguchi (1977), p. 238.
} 
From Eq. (34), we have $\lim _{\beta \rightarrow+\infty} \partial b_{2} / \partial \alpha=+\infty$ so that we have $b_{2}>1$ for sufficiently large $\alpha>0$ and $\beta>0$. This proves that Proposition 1 also applies to the system $\left(\mathrm{S}_{2}\right)$.

\section{NUMERICAL EXPERIMENTATION OF 'MODEL 1'}

Analytical approach by means of linear approximation of the system without stochastic disturbance which was developed in the previous sections gives us relatively little information on the behavior of the original nonlinear system with stochastic disturbance. Numerical approach will provide us some useful insight, which cannot be obtained if we stick to the analytical approach. In this section, we shall summarize the results of our numerical experimentation of 'model 1'.

We specify the functional forms of the relevant functions and the parameter values as follows:

$$
\begin{aligned}
& I\left(Y_{t}, K_{t}, r_{t}\right)=f\left(Y_{t}\right)-0.3 K_{t}-r_{t}+147 ; \\
& f\left(Y_{t}\right)=(80 / \pi) \operatorname{Arctan}\{(2.25 \pi / 20) \\
& \left.\times\left(Y_{t}-165 / 0.66\right)\right\}+35 \text {; } \\
& r_{t}=r\left(Y_{t}, M_{t}\right)=10 \sqrt{Y_{t}}-M_{t} ; \\
& J\left(Y_{t}, \bar{E}\right):=-0.3 Y_{t}+50 \\
& c=0.8, \quad \tau=0.2, \quad p=1, \quad r_{f}=6 ; \\
& c T_{0}+C_{0}+G=115 .
\end{aligned}
$$

The function $f\left(Y_{t}\right)$ in Eq. (36) represents the Kaldorian S-shaped investment function (see Fig. 1). Equation (37) is the LM equation which describes the equilibrium condition in the money market, and Eq. (38) is the current account function." Substituting Eqs. (35)-(40) into the

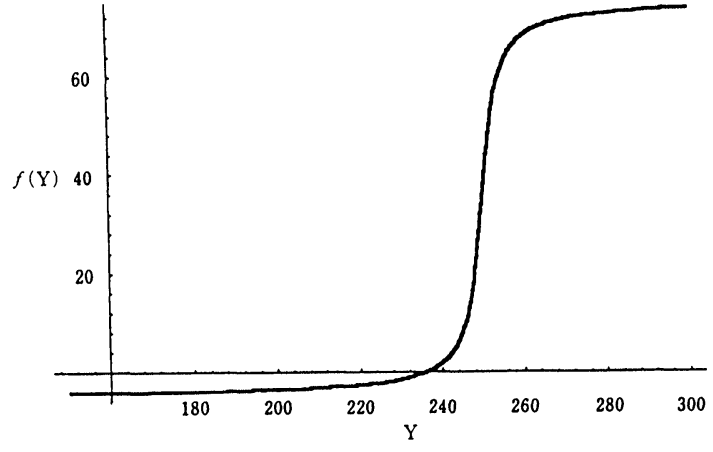

FIGURE 1

system $\left(S_{1}\right)$ in Section 2, we obtain the following expression:

(i) $Y_{t+1}-Y_{t}=\alpha\left\{-0.66 Y_{t}+f\left(Y_{t}\right)-0.3 K_{t}\right.$

$$
+147-10 \sqrt{Y_{t}}+M_{t}+165 \text {, }
$$

(ii)

$$
\begin{aligned}
& K_{t+1}-K_{t}=f\left(Y_{t}\right)-0.3 K_{t}-10 \sqrt{Y_{t}} \\
& +M_{t}+147 \text {, } \\
& \times\left(10 \sqrt{Y_{t}}-M_{t}-6\right) \text {. }
\end{aligned}
$$

In this system, it is assumed that the 'degree of capital mobility' $(\beta)$ is fluctuated by noise, and we select the parameters $\alpha$ and $\beta$ as the bifurcation parameters. $^{* *}$

If we assume that $\beta=1$ and $\sigma=0$, the equilibrium solution of the system (41) becomes $\left(Y^{*}, K^{*}, M^{*}\right)$ $\simeq(250,503,127)$. The equilibrium national income $Y^{*}$ is independent of the values of $\alpha$ and $\beta$. On the other hand, $K^{*}$ and $M^{*}$ depend on the values of $\alpha$ and $\beta$. Our numerical simulation shows that the behavior of this model can be very complex even if the noise does not exist $(\sigma=0)$, and the hidden structure of the system may be sometimes revealed rather than obscured when the system is fluctuated by noise.

\footnotetext{
${ }_{* *}^{\#}$ Note that under the system of fixed exchange rates $E$ is fixed, so that we need not explicitly introduce $E$ as a variable into the model. ${ }^{* *}$ In other words, in this model the noise effect is modeled by means of the 'parametric noise' rather than usual additive noise.
} 


\subsection{Dynamics of National Income}

In this subsection, we shall consider the dynamics of national income $(Y)$. First, let us consider the case without noise $(\sigma=0)$. Figures 2 and 3 are the bifurcation diagrams of national income with respect to the parameters $\alpha$ and $\beta$ respectively. "बा Figure 2 shows that the period of income fluctuation increases rapidly as the adjustment speed in the goods market $(\alpha)$ increases, and eventually the chaotic behavior emerges. However, as the

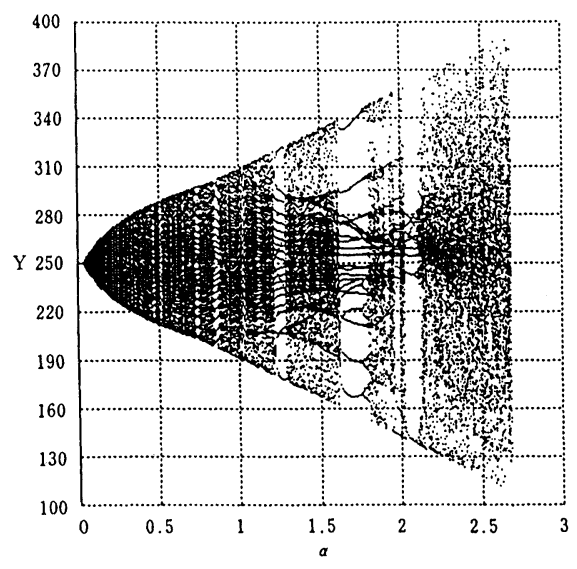

FIGURE 2 Bifurcation diagram of $Y$ without noise (parameter: $\alpha$ ).

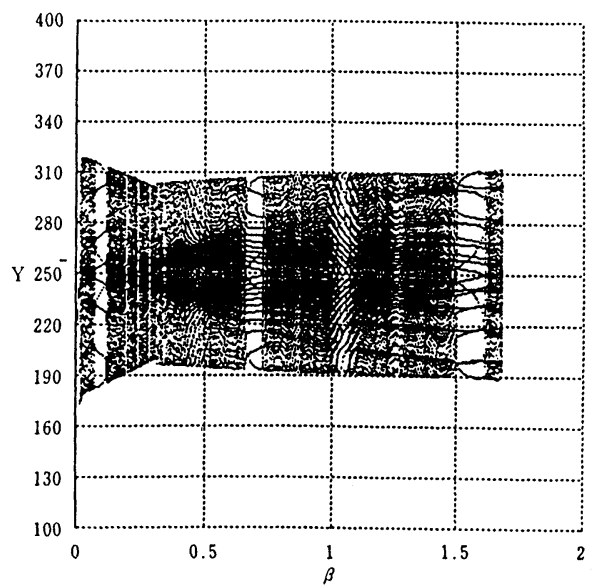

FIGURE 3 Bifurcation diagram of $Y$ without noise (parameter: $\beta$ ). parameter $\alpha$ increases furthermore, the 'window' which represents the periodical behavior emerges, and then the chaotic region reappears. We can confirm this statement by observing the largest Lyapunov exponent (see Fig. 4). Figures 5 and 6 are the bifurcation diagram and the largest Lyapunov exponent with small stochastic disturbance $(\sigma=0.01)$. We can see from these figures that the window of periodical solution disappears and

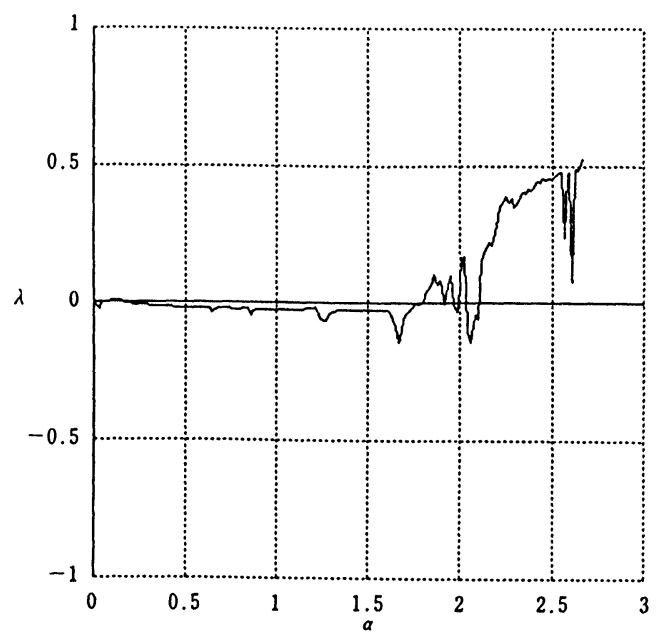

FIGURE 4 The largest Lyapunov exponent $(\lambda)$ without noise.

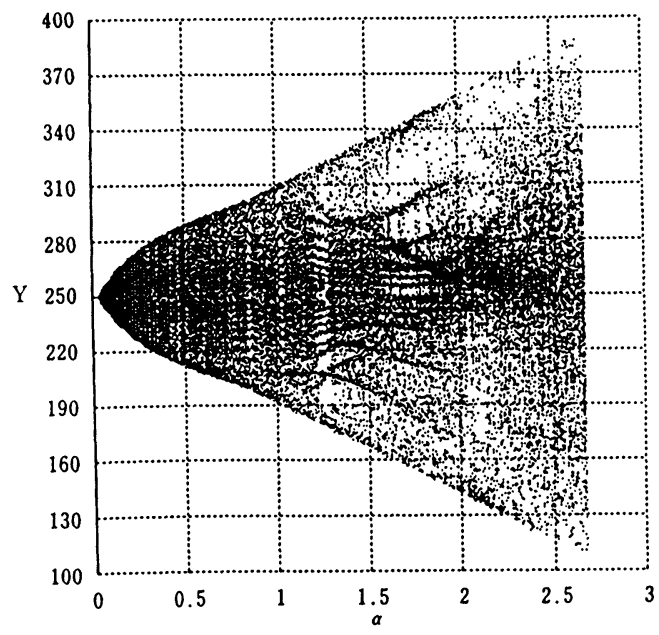

FIGURE 5 Bifurcation diagram of $Y$ with noise $(\sigma=0.01)$.

\footnotetext{
ฯ $\mathrm{It}$ is assumed that $\beta=1$ when $\alpha$ is selected as a bifurcation parameter, and $\alpha=1$ is assumed when $\beta$ is selected as a bifurcation parameter.
} 


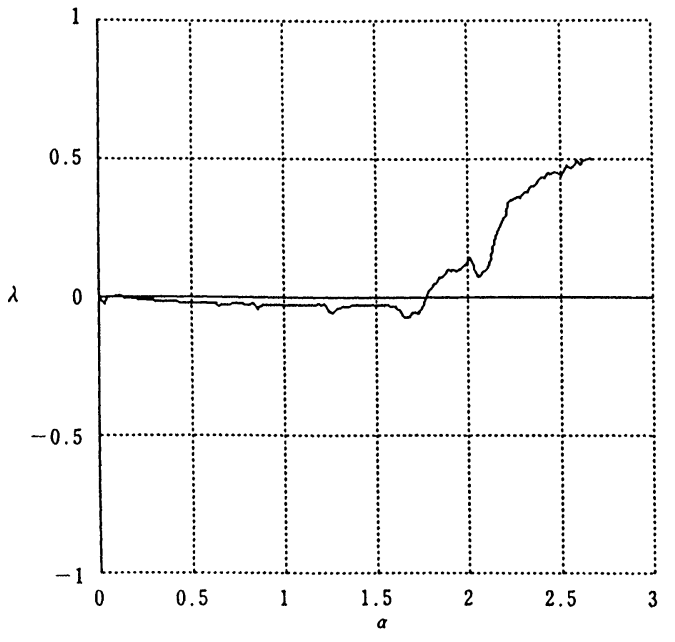

FIGURE 6 The largest Lyapunov exponent with noise $(\sigma=0.01)$.

the behavior of the system becomes more chaotic because of the noise effects.

Now, let us compare the system with noise effects and that without noise effects. Figures 2 and 4 show that the behavior of the system without noise is chaotic when $\alpha=2.0$, while the 'window' of the periodical solution appears when $\alpha=2$.1. However, the behavior of this system becomes chaotic again when $\alpha=2.2$. Figures 7-9 give the attractors of the system in $K-Y$ plane in these three cases. Figure 10 is the attractor of the system with small noise effects $(\sigma=0.01)$ when $\alpha=2.1$. The shape of the attractor in Fig. 10 is similar to that in Fig. 7 or 9. If there is no stochastic disturbance, the periodical trajectory is stable and chaotic trajectory is unstable at the 'window'. This implies that the chaotic structure is invisible and hidden at the 'window' if there is no stochastic disturbance. However, our numerical experimentation shows that the stochastic noise can make visible this hidden chaotic structure in some situations. Hence, it is not correct to say that the noise only obscures the basic structure of the system.

Figure 11 is the bifurcation diagram of $Y$ with respect to the parameter $\beta$ when $\beta$ is subject to the small stochastic disturbance $(\sigma=0.01)$. Also in this case, some 'windows' of the periodical solution

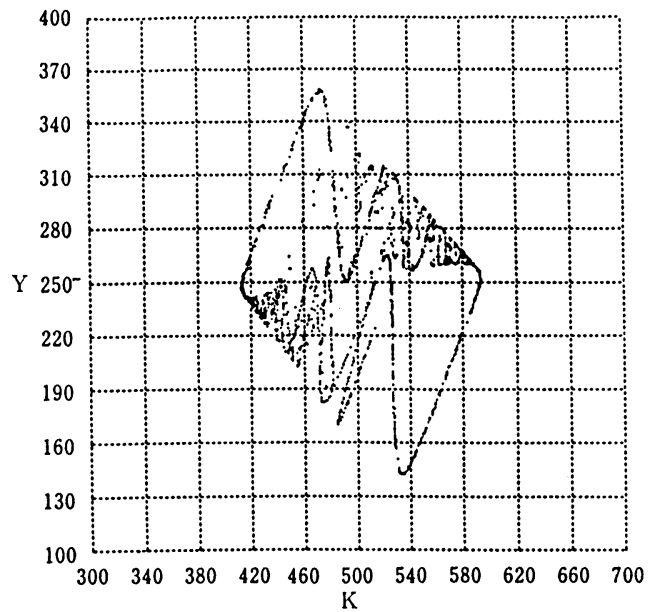

FIGURE 7 Attractor in $Y-K$ plane without noise when $\alpha=2.0$.

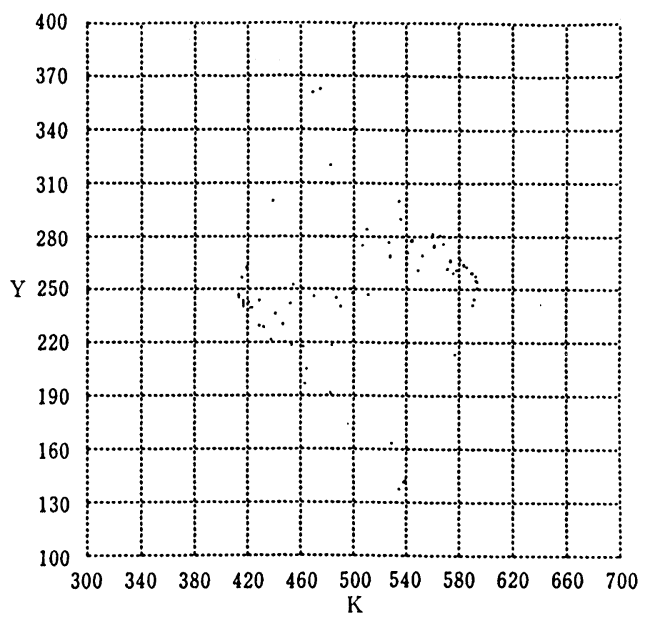

FIGURE 8 Attractor in $Y-K$ plane without noise when $\alpha=2.1$.

disappear because of the noise effects (compare Figs. 3 and 11).

\subsection{Dynamics of Capital Stock}

Dynamics of capital stock are given by Figs. 12-15. Figures 12 and 13 compare the bifurcation diagram of $K$ with respect to $\alpha$ without noise and that with small noise. Figures 14 and 15 are bifurcation diagrams of $K$ with respect to $\beta$. 


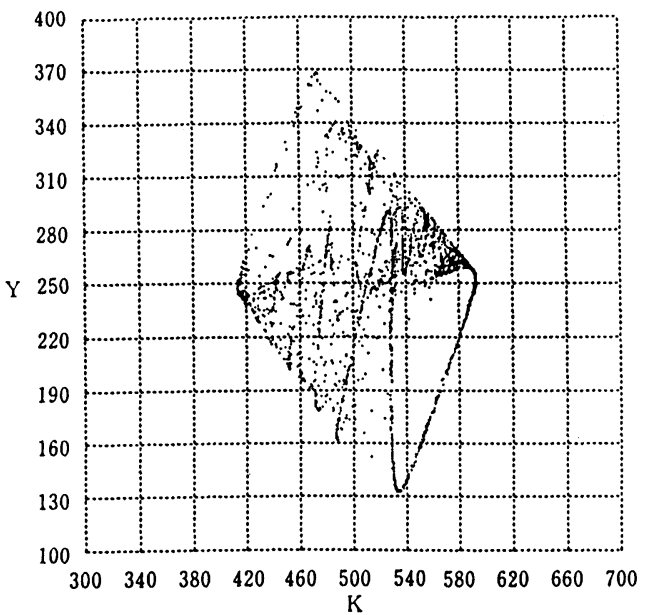

FIGURE 9 Attractor in $Y-K$ plane without noise when $\alpha=2.2$.

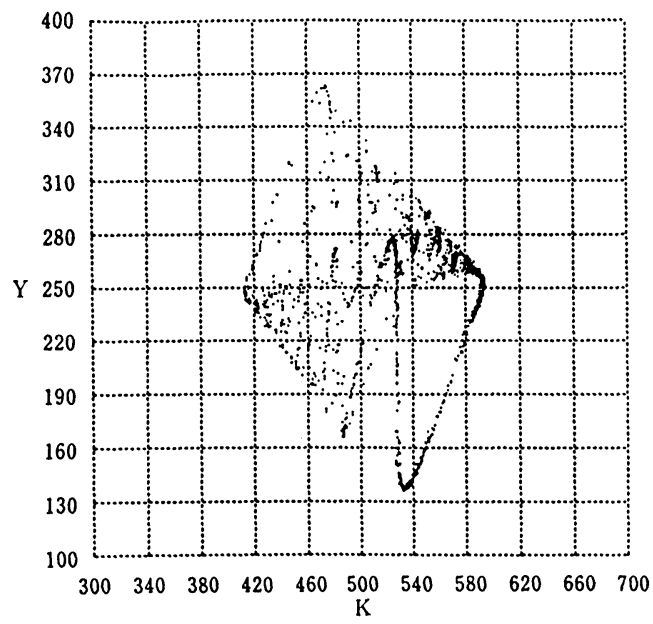

FIGURE 10 Attractor in $Y-K$ plane with noise when $\alpha=2.1$.

\section{NUMERICAL EXPERIMENTATION OF 'MODEL 2'}

We can construct a numerical example of 'model 2' by slightly modifying Eq. (41). In fact, we can obtain such a model by replacing $0.3 K_{t}$ in Eq. (41)(i) with zero and keeping other two equations of (41)(ii) and (iii) intact. However, this slight modification changes the behavior of the system considerably.

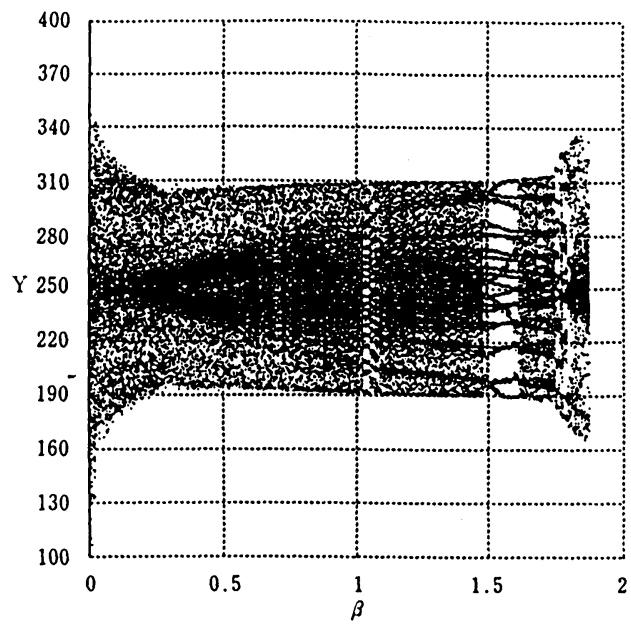

FIGURE 11 Bifurcation diagram of $Y$ with noise $(\sigma=0.01)$.

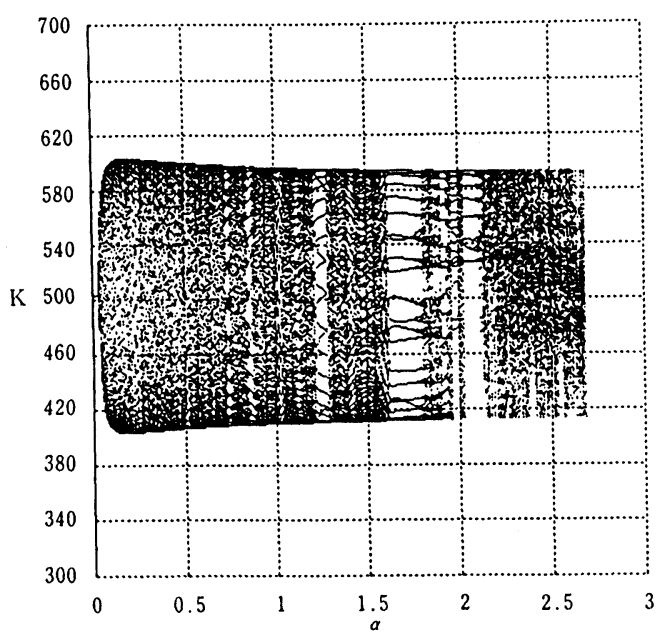

FIGURE 12 Bifurcation diagram of $K$ without noise (parameter: $\alpha$ ).

Figure 16 is the bifurcation diagram of $Y$ with respect to the changes of the parameter $\alpha$ without noise effects, and Fig. 17 shows the largest Lyapunov exponent in this case. The equilibrium point is stable when $\alpha$ is small, but it becomes unstable and two period cycle becomes stable when $\alpha$ exceeds 2.25. Then, the period-doubling bifurcations occur rapidly, and the behavior of the system becomes chaotic.

It is worth to note that this system has two equilibrium points. In fact, we obtained Fig. 16 by 


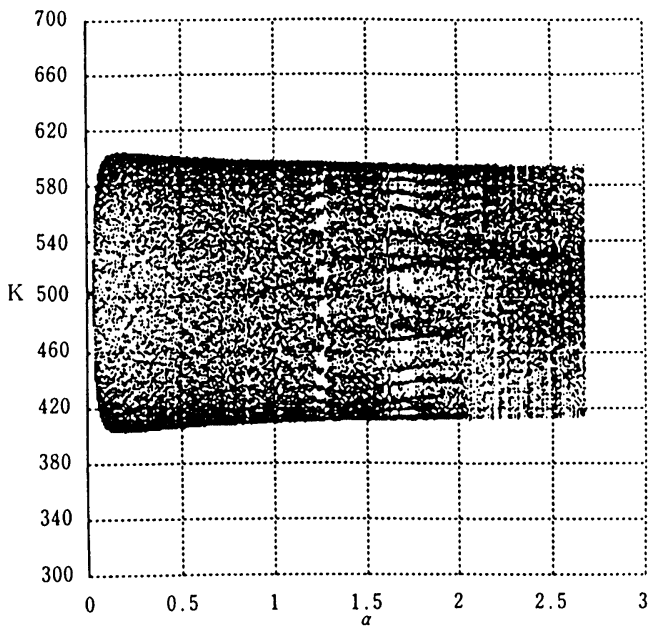

FIGURE 13 Bifurcation diagram of $K$ with noise (parameter: $\alpha)(\sigma=0.01)$.

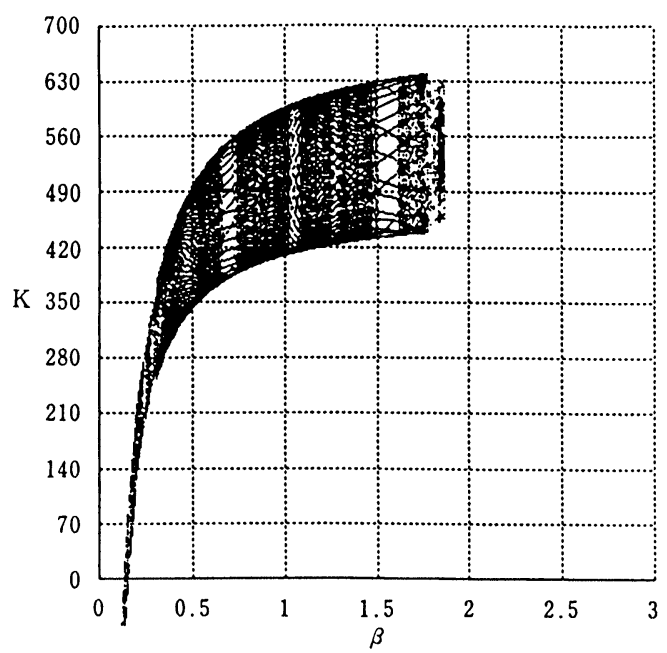

FIGURE 14 Bifurcation diagram of $K$ without noise (parameter: $\beta$ ).

adopting the initial condition $\left(Y_{0}, K_{0}, M_{0}\right)$, which is near from the equilibrium point of 'model 1', i.e. $\left(Y^{*}, K^{*}, M^{*}\right) \simeq(250,503,127)$. If we adopt another initial condition, we can obtain another attractor and another bifurcation diagram. However, Fig. 16 shows that the fusion of two attractors occurs so that the fluctuating area of $Y$ expands suddenly when $\alpha$ exceeds 2.5. Figure 17 shows that there are several 'windows' of periodic solutions in the area of $\alpha>2.5$.

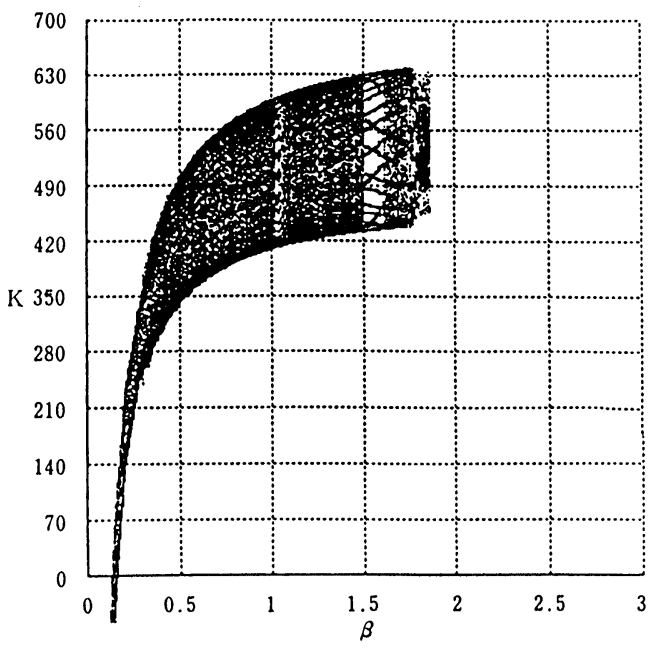

FIGURE 15 Bifurcation diagram of $K$ with noise (parameter: $\beta)(\sigma=0.01)$.

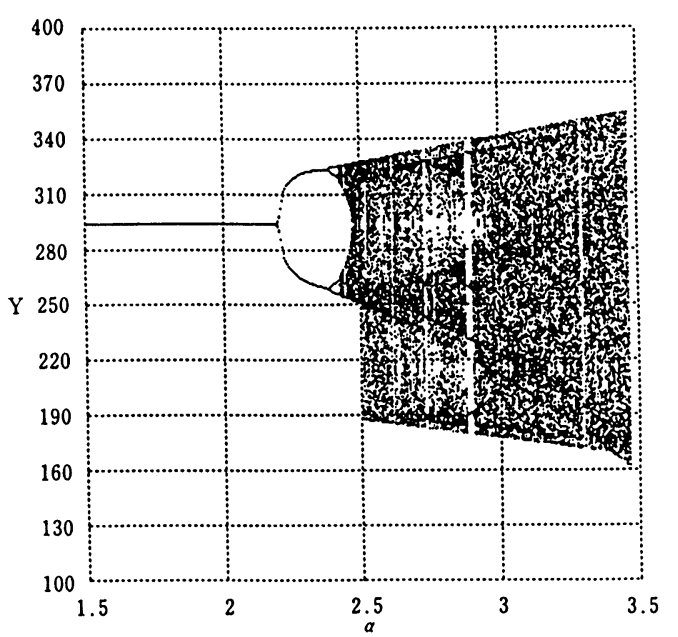

FIGURE 16 Bifurcation diagram of $Y$ without noise (parameter: $\alpha$ ).

Figure 18 is the bifurcation diagram which is fluctuated by noise $(\sigma=0.08)$. This figure shows that the 'windows' of the periodic solutions disappear because of the noise effect. Furthermore, in this figure the fusion of the attractors occur even if $\alpha<2.5$. We can interpret this phenomenon that the hidden structure of the system is revealed because of the noise effects. For convenience, let us say that the economy is in 'boom' when $Y_{t}>250$ and it is in 'slump' when $Y_{t}<250$. Comparing Figs. 16 and 18, 


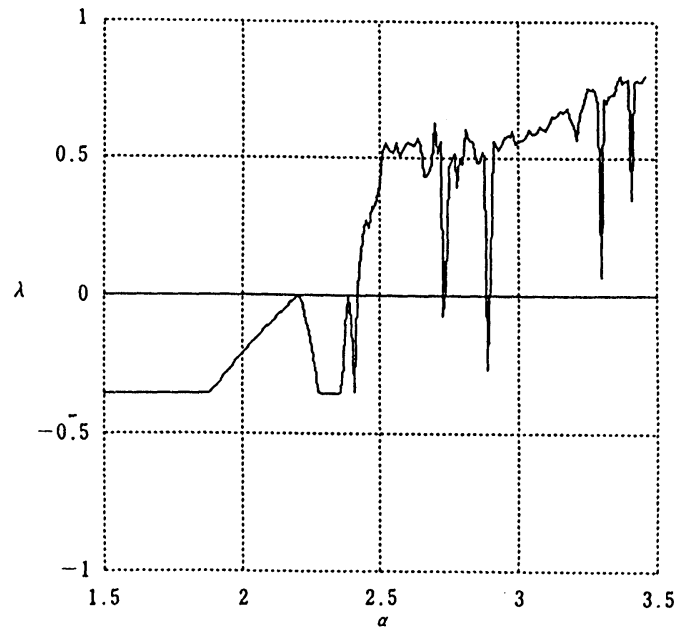

FIGURE 17 The largest Lyapunov exponent $(\lambda)$ without noise.

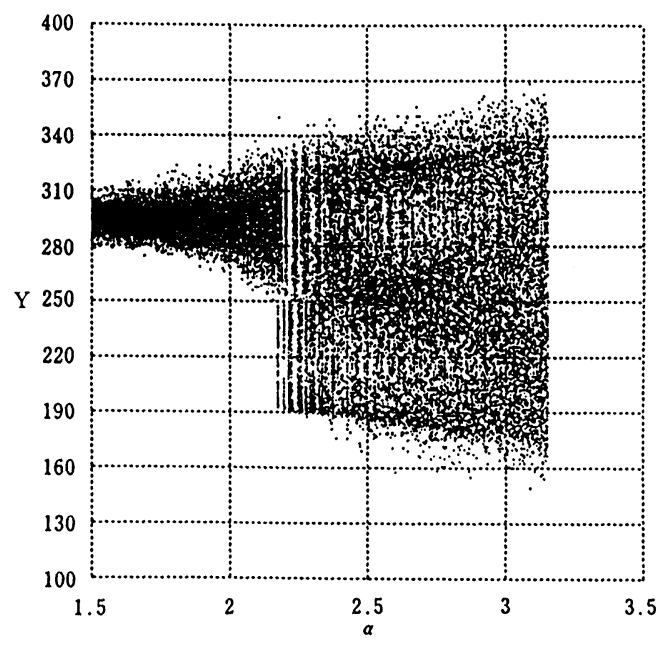

FIGURE 18 Bifurcation diagram of $Y$ with noise $(\sigma=0.08)$.

we can conclude that even if the economy is in boom, some stochastic disturbance can bring abut slump if the depressing structure is hidden in the system.

Figures 19 and 20 compare the trajectories of $Y_{t}$ without noise and that with noise in the case of $\alpha=2.5$. Figures 21 and 22 show the result of the similar experimentation in the case of $\alpha=2.55$. These examples show that the noise may transform the lasting booms into the violent alternations of booms and slumps, but the opposite case is also

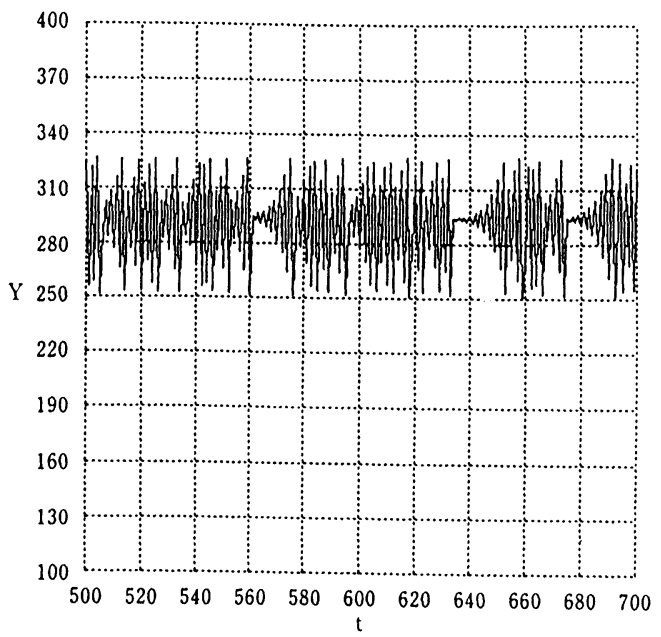

FIGURE 19 Trajectory of $Y$ without noise when $\alpha=2.5$.

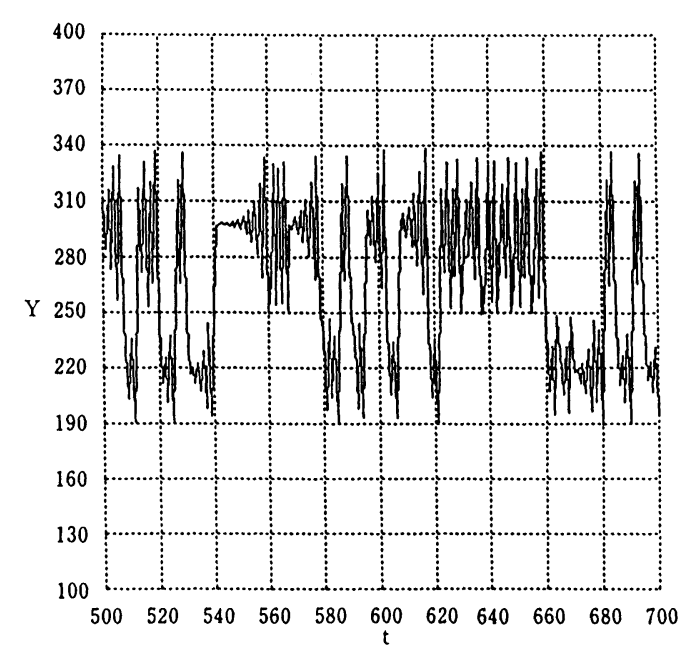

FIGURE 20 Trajectory of $Y$ with noise when $\alpha=2.5$ $(\sigma=0.08)$.

possible. This is one of the important lessons of our numerical simulations.

\section{COMPARISON WITH ASADA (1995)'S VERSION}

Before closing this paper, let us make a comparison between the model in this paper and Asada (1995)'s original version with continuous time without noise. Asada (1995)'s system of equations, which 


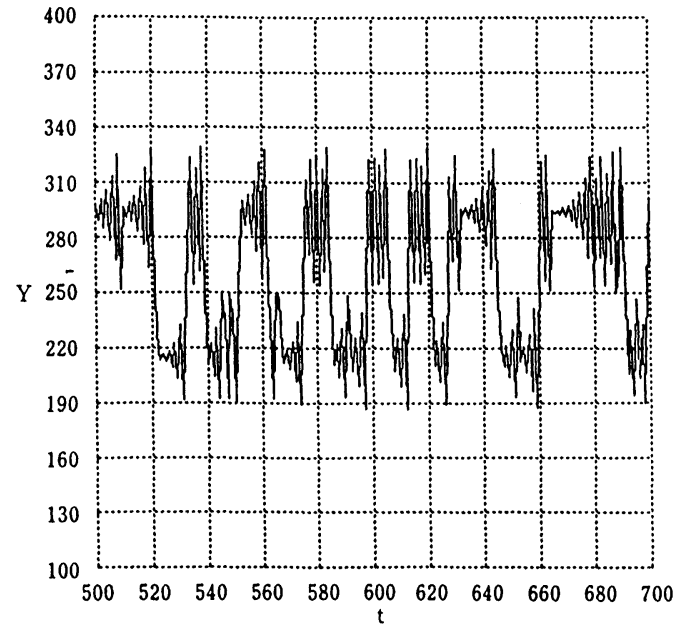

FIGURE $21 \quad$ Trajectory of $Y$ without noise when $\alpha=2.55$.

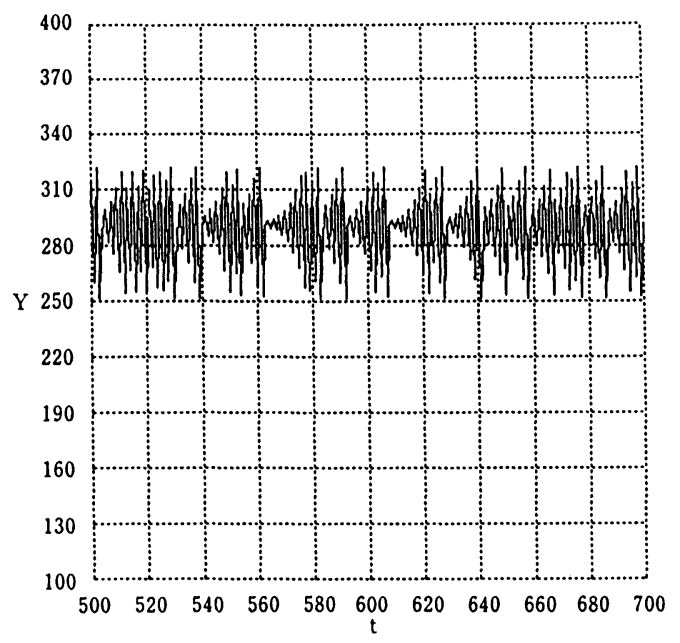

FIGURE 22 Trajectory of $Y$ with noise when $\alpha=2.55$ $(\sigma=0.08)$.

corresponds to Eq. $\left(\mathrm{S}_{1}\right)$ in this paper, is given as follows:

(i)

$$
\begin{aligned}
\mathrm{d} Y / \mathrm{d} t= & \alpha\left[c(1-\tau) Y+c T_{0}\right. \\
& +C_{0}+G+I(Y, K, r(Y, M)) \\
& +J(Y, \bar{E})-Y] \\
\equiv & f_{1}(Y, K, M),
\end{aligned}
$$

(ii) $\mathrm{d} K / \mathrm{d} t=I(Y, K, r(Y, M))$

$$
\equiv f_{2}(Y, K, M)
$$

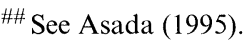

(iii)

$$
\begin{aligned}
\mathrm{d} M / \mathrm{d} t & =p J(Y, \bar{E})+\beta p\left\{r(Y, M)-r_{f}\right\} \\
& \equiv f_{3}(Y, M ; \beta) .
\end{aligned}
$$

Asada (1995) derived the following results analytically under some assumptions:

(1) The equilibrium point of the system $S_{1}^{\prime}$ is locally stable if $\beta>0$ is sufficiently small, and it becomes a saddle point if $\beta$ is sufficiently large.

(2) There exists the parameter value $\beta_{0}>0$ at which the Hopf bifurcation occurs. In other words, there exist some nonconstant periodic solutions at some values of $\beta$ which is sufficiently close to $\beta_{0}$.

Asada (1995) also presented some numerical simulations which support the above analytical results. However, Asada (1995)'s original version could not produce chaotic motion, but it produced rather 'regular' movement. Compared to Asada (1995)'s version, the discrete time version with and without noise which is presented in this paper can produce much complex and richer behavior, and it provides us a foundation to further research.

\section{CONCLUDING REMARKS}

In this paper, we investigated the discrete time version of the Kaldorian business cycle model in an open economy with and without noise effects by means of analytical method and numerical simulations. As a result, we could find some interesting behaviors of the system including chaotic movement. However, the model which was presented in this paper is restricted to the system of fixed exchange rates. While in the system of fixed exchange rates the money supply becomes an endogenous variable, in the system of flexible exchange rates we can consider the money supply as the exogenous variable which is controlled by the central bank. \#\# Obviously, the next step must be the analysis of the system of flexible exchange rates. This is the theme which we shall study in another paper. 


\section{Acknowledgment}

An earlier version of this paper was presented at the First International Conference on DCDNS (Discrete Chaotic Dynamics in Nature and Society) which was held at Beer-Sheva, Israel (October 21, 1998). This research was financially supported by Chuo University Grant for Special Research, Waseda University Grant for Special Research Projects No. 98A-074, and Grant-in-Aid for Scientific Research No. 09640285 from the Ministry of Education, Science and Culture of Japanese Government.

\section{References}

Asada, T. (1995): "Kaldorian dynamics in an open economy." Journal of Economics/Zeitschrift für Nationalokönomie 62, $239-269$.
Chang, W.W and Smyth, D.J. (1971): "The existence and persistence of cycles in a nonlinear model: Kaldor's 1940 model re-examined." Review of Economic Studies 38, 37-44.

Dohtani, A., Misawa, T., Inaba, T., Yokoo, M. and Owase, T. (1996): "Chaos, complex transients and noise: Illustrations with a Kaldor model." Chaos, Solitons and Fractals 7, $2157-$ 2174.

Gabisch, G. and Lorenz, H.W. (1989): Business Cycle Theory (2nd edn.) Berlin, Heidelberg, New York and Tokyo: SpringerVerlag.

Gandolfo, G. (1996): Economic Dynamics (3rd edn.) Berlin, Heidelberg, New York and Tokyo: Springer-Verlag.

Kaldor, N. (1940): "A model of the trade cycle." Economic Journal 50, 78-92.

Kosobud, R.F. and O'Nell, W.D (1972): "Stochastic implications of orbital asymptotic stability of nonlinear trade cycle model." Econometrica 40, 69-86.

Lorenz, H.W. (1993): Nonlinear Dynamical Economics and Chaotic Motion. (2nd edn.) Berlin, Heidelberg, New York and Tokyo: Springer-Verlag.

Okuguchi, K. (1977): Mathematical Foundations for Economic Analysis. Tokyo: McGraw-Hill Kogakusha (in Japanese). 


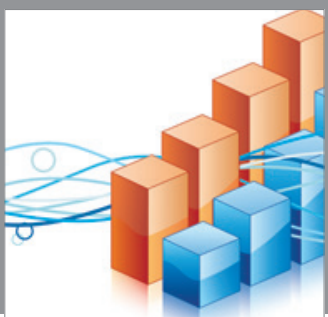

Advances in

Operations Research

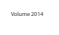

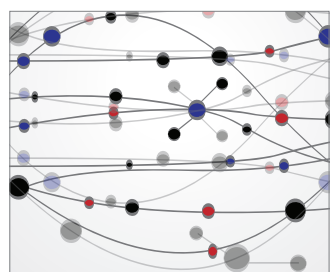

\section{The Scientific} World Journal
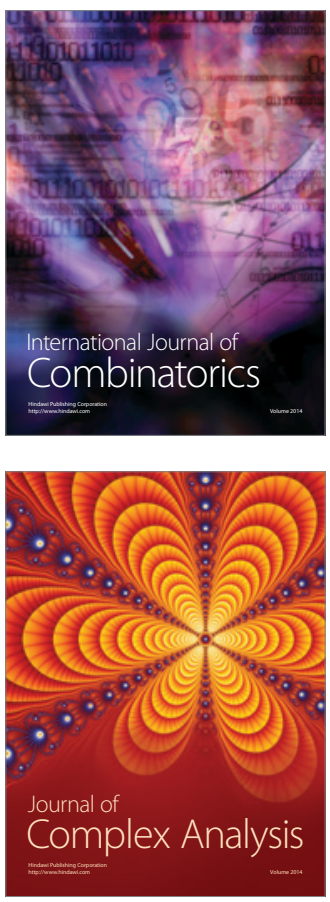

International Journal of

Mathematics and

Mathematical

Sciences
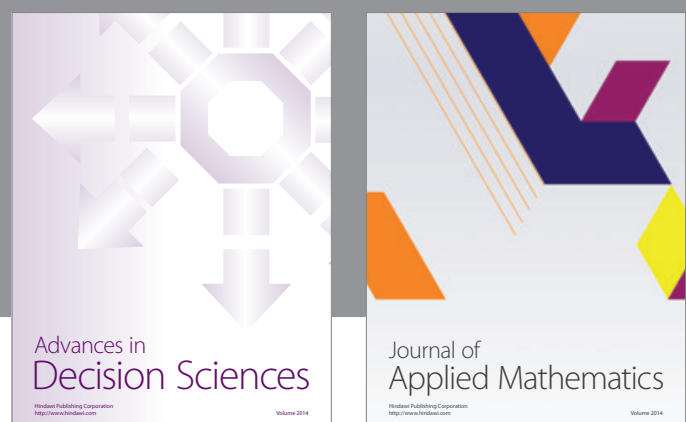

Journal of

Applied Mathematics
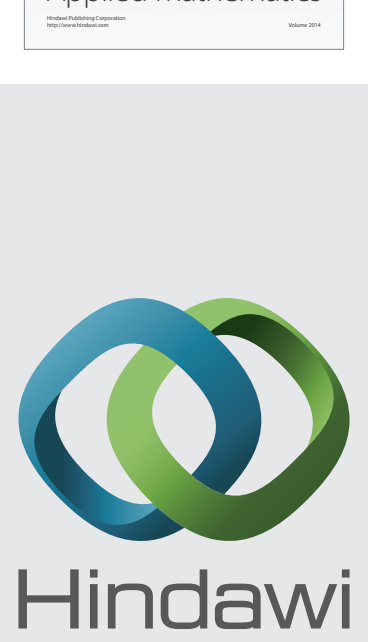

Submit your manuscripts at http://www.hindawi.com
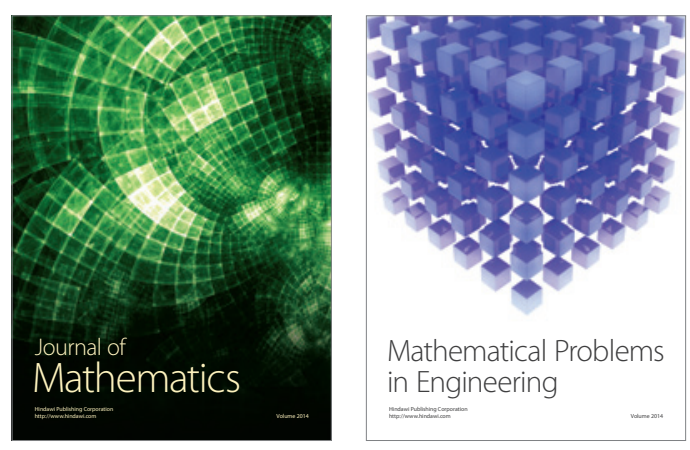

Mathematical Problems in Engineering
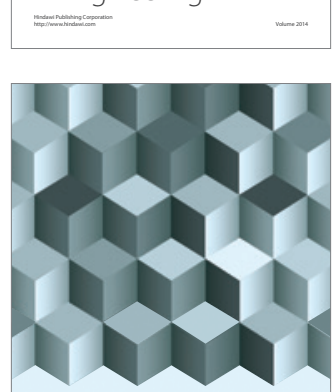

Journal of

Function Spaces
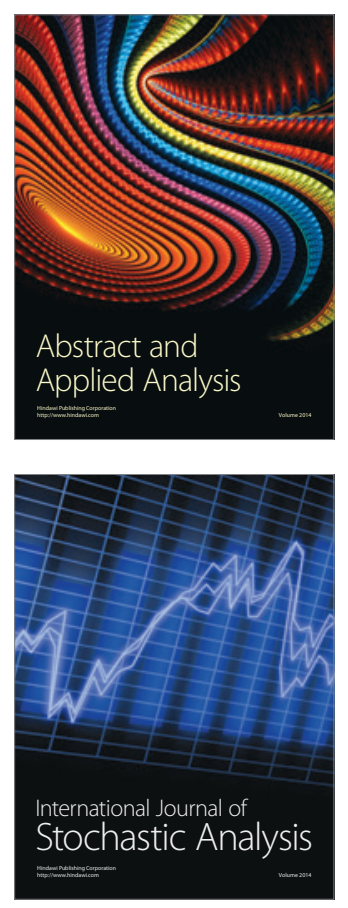

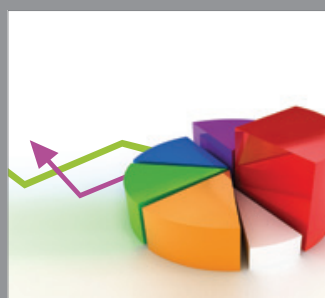

ournal of

Probability and Statistics

Promensencen
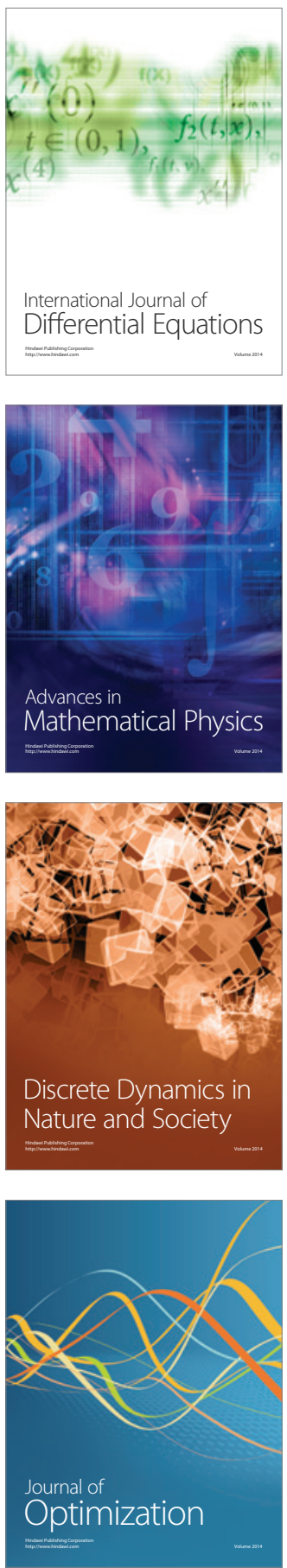